\title{
The Linearity of the Euler Equation as a Result of the Compressibility of a Fluid
}

\author{
Vladimir Kirtskhalia ${ }^{1,2}$ \\ ${ }^{1}$ I. Vekua Sokhumi Institute of Physics and Technilogy (SIPT), Tbilisi, Georgia \\ ${ }^{2}$ Sokhumi State University, Tbilisi, Georgia \\ Email: sipt@sipt.org, info@sou.edu.ge
}

How to cite this paper: Kirtskhalia, V. (2019) The Linearity of the Euler Equation as a Result of the Compressibility of a Fluid. Journal of Modern Physics, 10, 452-458. https://doi.org/10.4236/jmp.2019.104030

Received: January 20, 2019

Accepted: March 16, 2019

Published: March 19, 2019

Copyright $\odot 2019$ by author(s) and Scientific Research Publishing Inc. This work is licensed under the Creative Commons Attribution International License (CC BY 4.0).

http://creativecommons.org/licenses/by/4.0/

\begin{abstract}
It is shown that when the compressibility of a fluid is taken into account, the nonlinear term disappears in the Euler equation. The validity of this approach is proved by the example of capillary waves.
\end{abstract}

\section{Keywords}

Euler Equation, Mass Continuity Equation, Fluid Compressibility, Capillary Waves

\section{Introduction}

In the monography [1], the expression for the square of phase speed of capillary wave on the surface of fluid is received, according to which it generally depends on its depth and consists of the sum of two members. One of them defines the influence of gravitational field, and the second the influence of surface tension, and as a result of which this wave is called capillary-gravitational. This result was obtained under the assumption of the potentiality of motion and incompressibility of a fluid, on which the entire existing theory of gravitational waves is based [2]-[15]. In the work [16] it was shown that the movement of fluid in a gravitational field of the Earth cannot be potential. It was also shown that the condition of incompressibility is inapplicable to liquids, because it leads to the existence of an internal wave [1], the nature of which is completely inexplicable from the point of view of physics. This conclusion was made on the basis of a correct determination of the speed of sound [17] and a new definition of the criteria of compressibility and incompressibility of the medium [18]. This situation made it necessary to refine mass conservation equation, and after its refinement, the gravity acceleration disappeared from the dispersion equation of capil- 
lary-gravitational wave, and it becomes purely capillary [16]. The further investigations of gas and hydrodynamics have shown that Euler equation (fluid motion equation) also must be subject to refinement, since for it assumes that the density of a fluid is constant. This approach leads to the fact that the nonlinear term disappears from this equation and it becomes linear.

In this work, we apply Euler's refined equation towards capillary waves and show that despite the use of the boundary condition at the bottom of the liquid, the depth of the reservoir disappears from the dispersion equation, and thus the phase velocity of the capillary wave depends only on its length. In addition, it will be shown that there is no stability condition for a capillary wave, i.e. it is always stable in the first equation of system (72) in [16] typo, which the interested reader can easily correct.

\section{Improved Euler Equation}

In existing gas and hydrodynamics theory, Euler equation is applied in the form:

$$
\rho \frac{\mathrm{d} \boldsymbol{V}}{\mathrm{d} t}=\rho\left[\frac{\partial \boldsymbol{V}}{\partial t}+(\boldsymbol{V} \nabla) \boldsymbol{V}\right]=-\nabla P+\rho \boldsymbol{g}
$$

where: $\rho$-density, $\boldsymbol{V}$-speed fluid particles, $P$-pressure, $\boldsymbol{g}$-gravitational acceleration. Here supposed to that liquid is incompressible and consequently $\rho=$ const . In several works (see e.g. [19]), it was shown by us, that updated continuity equation of mass has the following form:

$$
\frac{\partial \rho}{\partial t}+(\boldsymbol{V} \nabla) \rho=-\rho \nabla \boldsymbol{V}-\frac{\boldsymbol{V} \nabla P}{C_{p}^{2}}
$$

where $C_{p}$ is isobaric sound speed in the liquid which can be considered as infinitely large, and we have:

$$
\frac{\mathrm{d} \rho}{\mathrm{d} t}=\frac{\partial \rho}{\partial t}+(\boldsymbol{V} \nabla) \rho=-\rho \nabla \boldsymbol{V}
$$

From (3) this it follows that liquid is compressible medium $(\nabla \boldsymbol{V} \neq 0)$ end $\mathrm{d} \rho / \mathrm{d} t \neq 0$. Consequently, Euler equation should be written in the form of:

$$
\frac{\mathrm{d}(\rho \boldsymbol{V})}{\mathrm{d} t}=\boldsymbol{V} \frac{\mathrm{d} \rho}{\mathrm{d} t}+\rho \frac{\mathrm{d} \boldsymbol{V}}{\mathrm{d} t}=-\nabla P+\rho \boldsymbol{g}
$$

Then if we substitute $\mathrm{d} \rho / \mathrm{d} t$ from (3) in (4), we get:

$$
\begin{aligned}
& -\boldsymbol{V} \rho \nabla \boldsymbol{V}+\rho \frac{\mathrm{d} \boldsymbol{V}}{\mathrm{d} t}=-\nabla P+\rho \boldsymbol{g} \\
& \Rightarrow \rho\left[\frac{\mathrm{d} \boldsymbol{V}}{\mathrm{d} t}-(\boldsymbol{V} \nabla) \boldsymbol{V}\right]=-\nabla P+\rho \boldsymbol{g} \\
& \Rightarrow \rho \frac{\partial \boldsymbol{V}}{\partial t}=-\nabla P+\rho \boldsymbol{g}
\end{aligned}
$$

So, we have a system of two equations: 


$$
\left\{\begin{array}{l}
\rho \frac{\partial \boldsymbol{V}}{\partial t}=-\nabla P+\rho \boldsymbol{g} \\
\frac{\partial \rho}{\partial t}+(\boldsymbol{V} \nabla) \rho=-\rho \nabla \boldsymbol{V}
\end{array}\right.
$$

We see that the nonlinear term from the Euler equation has disappeared. It remains only in the mass conservation equation.

\section{Dispersion Equation of Capillary Waves and Her Decision}

Let's presenting all the variables from the system (6) in the form of the sum of their stationary and perturbed values $f=f_{0}+f^{\prime}$ and supposing that $f^{\prime} / f_{0}<1$, after linearization it will take the following form:

$$
\left\{\begin{array}{l}
\rho_{0} \frac{\partial V}{\partial t}=-\nabla P+\frac{\boldsymbol{g}}{C^{2}} P \\
\frac{1}{C^{2}}\left(\frac{\partial P}{\partial t}+\boldsymbol{V}_{0} \nabla P\right)=-\rho_{0} \nabla \boldsymbol{V}
\end{array}\right.
$$

where we used: state equation of medium $\rho^{\prime}=P^{\prime} / C^{2} \quad$ ( $C$-adiabatic speed of sound in the liquid), liquid equilibrium equation $\nabla P_{0}=\rho_{0} g$ and perturbed values don't have stokes.

If $\bar{V}_{0}$ there is a stationary velocity of fluid flow, then the linearized Equation (1) will be

$$
\rho_{0}\left[\frac{\partial \boldsymbol{V}}{\partial t}+\left(\boldsymbol{V}_{0} \nabla\right) \boldsymbol{V}\right]=-\nabla P+\frac{\boldsymbol{g}}{C^{2}} P
$$

As we see, this equation, which determines the acceleration of a liquid particle, contains a stationary velocity of motion, on which the acceleration should not depend. This fact irrefutably proves the validity of formula (4).

Appling to the first equation of system (7) operator $\nabla$ and to the second operator $\partial / \partial t$, they easily reduced to differential equation for perturbed pressure

$$
\Delta P-\frac{1}{C^{2}}\left(\vec{g}+\vec{V}_{0} \frac{\partial}{\partial t}\right) \nabla P-\frac{1}{C^{2}} \frac{\partial^{2} P}{\partial t^{2}}=0
$$

Presenting now the perturbed pressure in the form of periodic function

$$
P(x, z, t)=P_{a}(z) \exp [i(k x-\omega t)]
$$

and supposing that $\boldsymbol{V}_{0}=\boldsymbol{e}_{x} V_{0}$ and $\boldsymbol{g}=-\boldsymbol{e}_{z} g$, we will get ordinary differential equation of second order for the amplitude of pressure disturbance in the following form:

$$
\frac{\mathrm{d}^{2} P_{a}(z)}{\mathrm{d} z^{2}}+\frac{g}{C^{2}} \frac{\mathrm{d} P_{a}(z)}{\mathrm{d} z}+\left[\frac{\omega}{C^{2}}\left(\omega-k V_{0}\right)-k^{2}\right] P_{a}(z)=0
$$

The Equation (10) describes pressure disturbance on both sides of surface of tangential discontinuity $z=0$. We solve this equation for air $(z>0)$ in the form: 


$$
P_{a 1}(z)=A \exp (\gamma z),
$$

where upon, taking into account the attenuation of the disturbance when $z \rightarrow \infty$, for $\gamma$ we will get:

$$
\gamma=-\frac{k}{\theta_{1}}\left\{1+\sqrt{1+\theta_{1}^{2}\left[1-\frac{U_{p}\left(U_{p}-V_{0}\right)}{C_{1}^{2}}\right]}\right\}<0
$$

where $C_{1}$ is sound speed in the air on the sea level, $U_{p}=\omega / k$ is phase speed of surface wave and $\theta_{1}$ is dimensionless value and it is equal to

$$
\theta_{1}=\frac{2 k C_{1}^{2}}{g}
$$

For the liquid $(z<0)$, because of its depth limitation, from (10) analogically we will have:

$$
P_{a 2}(z)=B_{1} \exp \left(\delta_{1} z\right)+B_{2} \exp \left(\delta_{2} z\right)
$$

where

$$
\begin{gathered}
\delta_{1}=-\frac{k}{\theta_{2}}\left[1+\sqrt{1+\theta_{2}^{2}\left(1-\frac{U_{p}^{2}}{C_{2}^{2}}\right)}\right]<0 \\
\delta_{2}=-\frac{k}{\theta_{2}}\left[1-\sqrt{1+\theta_{2}^{2}\left(1-\frac{U_{p}^{2}}{C_{2}^{2}}\right)}\right]>0 \\
\theta_{2}=\frac{2 k C_{2}^{2}}{g}
\end{gathered}
$$

Let us denote liquid surface displacement along the axis $z$ through

$$
\xi(x, t)=a \exp [i(k x-\omega t)]
$$

and in this case, the boundary conditions on the surface $(z=0)$ and on the bottom $(z=-h)$ of the liquid will have the form of:

$$
\left\{\begin{array}{l}
\left.\left(P_{2}-P_{1}\right)\right|_{z=0}=-\alpha \frac{\partial^{2} \xi}{\partial x^{2}} \\
\left.V_{z 1}\right|_{z=0}=\frac{\partial \xi}{\partial t}+V_{0} \frac{\partial \xi}{\partial x} \\
\left.V_{z 2}\right|_{z=0}=\frac{\partial \xi}{\partial t} \\
\left.V_{z 2}\right|_{z=-h}=0
\end{array}\right.
$$

where $\alpha$ is the coefficient of surface tension.

Let's present perturbation velocity in the form of periodical function:

$$
\boldsymbol{V}(x, z, t)=\boldsymbol{V}_{a}(z) \exp [i(k x-\omega t)]
$$

and denote its $z$ component through disturbance pressure from the first equation of the system (7): 


$$
V_{z}(x, z, t)=-\frac{i}{\rho_{0} \omega}\left[\frac{\partial P_{a}(z)}{\partial z}+\frac{g}{C^{2}} P_{a}(z)\right] \exp [i(k x-\omega t)]
$$

Substituting (9), (11), (14) and (21) in the boundary conditions (19), we will get the system of homogenous equations referred to unknown coefficients $A, B_{1}, B_{2}$ and $a$ :

$$
\left\{\begin{array}{l}
A-B_{1}-B_{2}+\alpha k^{2} a=0 \\
\frac{\gamma+g / C_{1}^{2}}{\rho_{01} \omega} A-\left(\omega-k V_{0}\right) a=0 \\
\frac{\delta_{1}+g / C_{2}^{2}}{\rho_{02} \omega} B_{1}+\frac{\delta_{2}+g / C_{2}^{2}}{\rho_{02} \omega} B_{2}-\omega a=0 \\
\frac{\delta_{1}+g / C_{2}^{2}}{\rho_{02} \omega} \exp \left(-\delta_{1} h\right) B_{1}+\frac{\delta_{2}+g / C_{2}^{2}}{\rho_{02} \omega} \exp \left(-\delta_{2} h\right) B_{2}=0
\end{array}\right.
$$

Equating the determinant of the system (22) to zero, we will get dispersion relation for the wave on the liquid surface taking into account surface tension force in the form of:

$$
\begin{aligned}
& \frac{\delta_{1} \delta_{2}}{\rho_{02} \omega}\left(\omega-k V_{0}-\frac{\gamma}{\rho_{01} \omega} \alpha k^{2}\right)\left[\exp \left(-\delta_{1} h\right)-\exp \left(-\delta_{2} h\right)\right] \\
& -\frac{\gamma}{\rho_{01}}\left[\delta_{2} \exp \left(-\delta_{1} h\right)+\delta_{1} \exp \left(-\delta_{2} h\right)\right]=0
\end{aligned}
$$

Taking into account that on the sea level $C_{1} \cong 340 \mathrm{~m} / \mathrm{sec}$, let's consider the inequality

$$
\theta_{1}=\frac{2 k C_{1}^{2}}{g}>1 \Rightarrow k>\frac{g}{2 C_{1}^{2}} \Rightarrow \lambda<\frac{4 \pi C_{1}^{2}}{g}=1.45 \times 10^{5} \mathrm{~m}
$$

We can see, that to this inequality satisfies with the entire range of lengths of surface waves on the water, from capillary to tsunami. It is apparent that for the capillary waves length of which does not exceed a few centimeters, we have: $\theta_{2} \gg \theta_{1} \gg 1$. Considering also that $U_{p}^{2} / C_{2}^{2} \ll U_{p}^{2} / C_{1}^{2} \ll 1$, from (12), (15) and (16) we find $\gamma=\delta_{1}=-k, \delta_{2}=k$ and then neglecting $\rho_{01}$ with respect to $\rho_{02}$, the dispersion Equation (23) takes the form:

$$
\rho_{02} U_{p}^{2}+\rho_{01} V_{0} U_{p}-\alpha k=0
$$

the solution of which is

$$
U_{p}=\frac{-\rho_{01} V_{0} \pm \sqrt{\rho_{01}^{2} V_{0}^{2}+4 \alpha k \rho_{02}}}{2 \rho_{02}}
$$

\section{Discussion of Results}

In order to show the truthfulness of our results, let's consider earlier results and show their drawbacks. As it was said in the introduction, in the monography [1] dispersion relation for the capillary-gravitational wave on the surface of incompressible liquid is presented in the form of: 


$$
U_{p}^{2}=\left(\frac{\omega}{k}\right)^{2}=\left(\frac{g}{k}+\frac{\alpha k}{\rho_{02}}\right) \operatorname{th}(k h)
$$

from which it follows that when

$$
\frac{g}{k}>\frac{\alpha k}{\rho_{02}} \Rightarrow k<\sqrt{\frac{\rho_{02} g}{\alpha}} \Rightarrow \lambda>2 \pi \sqrt{\frac{\alpha}{\rho_{02} g}}=1.72 \mathrm{sm}
$$

the influence of the surface tension force is negligible, and the wave becomes purely gravitational. This conclusion contradicts to the classical experiment, in which a steel needle does not sink in a glass filled with water to the brim. This is because although the diameter of glass greatly exceeds the above specified length, the force of surface tension acts which balances the pressure produced by the needle. Thus, the dependence of phase speed on gravitational acceleration is excluded and consequently there is no existing condition that limits the length of capillary wave. Such a conclusion is quite understandable from the point of view of physics, because surface tension arises due to the interaction forces between molecules on the surface of a liquid that significantly exceed the gravitational force.

The contradiction associated with the influence of the gravitational field is eliminated by taking into account the compressibility of the fluid in the mass continuity equation. The solution of the problem for such a case is given in [17], where the dispersion equation is obtained in the form:

$$
U_{p}=\frac{\omega}{k}=\frac{\rho_{01} V_{0} \operatorname{th}(k h) \pm\left\{\operatorname{th}(k h)\left[\rho_{02} \alpha k-\rho_{01} \rho_{02} V_{0}^{2}\right]\right\}^{1 / 2}}{\rho_{02}}
$$

from which follows the condition of stability of capillary wave:

$$
V_{0} \leq \sqrt{\frac{\alpha k}{\rho_{01}}}
$$

From (29) it is easy to calculate that the wind with the speed of $V_{0}=5 \mathrm{~m} / \mathrm{s}$, will blow off capillary waves whose length $\lambda>1.6 \mathrm{~cm}$. However, simple observations show that capillary waves exist at quite stronger winds. In addition, since the capillary wave is a purely surface phenomenon, its phase speed must not depend on the depth of the fluid.

As it is apparent in the Equation (25), this contradiction is eliminated if in Euler equation, we consider liquid as compressible. Capillary wave is stable in any wind, if only the wind force does not exceed intermolecular interaction force and in this case, setting of the problem becomes meaningless. We can also see that phase speed of capillary wave does not depend on the depth of fluid.

\section{Conclusion}

Contradictions that are present in the theory of surface waves are described in detail in the works [16] and [19]. In this work, through the example of capillary waves we have explicitly investigated the causes of these contradictions and showed how to overcome them. We can say with confidence that our recom- 
mendations will result in overcoming contradictions not only in the theory of capillary waves but also in the theory of gravity waves too.

\section{Conflicts of Interest}

The author declares no conflicts of interest regarding the publication of this paper.

\section{References}

[1] Landau, L.D. and Lifshitz, E.N. (1988) Hydrodynamics, Vol. 6, Nauka, Moscow.

[2] Stoker, J.J. (1957) Water Waves. Intersciense Publisher Inc., New York.

[3] Whitham, G. (1974) Linear and Nonlinear Waves. John Wiley and Sons, Hoboken.

[4] Kowalik, Z. (2012) Introduction to Numerical Modeling of Tsunami Waves. Institute of Marine Science University of Alaska, Fairbank.

[5] Hassan, J.M., Mohamed, T.A., Mohammed, W.S. and Alawee, W.H. (2014) Journal of Fluids, 2014, Article ID: 325259.

[6] Chand, R. and Rana, G.C. (2014) Journal of Fluids, 2014, Article ID: 479107.

[7] Rabby, M.G., Shupti, S.P. and Molla, M.M. (2014) Journal of Fluids, 2014, Article ID: 757902. https://doi.org/10.1155/2014/757902

[8] Eldabe, N.T.M., Agoor, B.M. and Alame, H. (2014) Journal of Fluids, 2014, Article ID: 525769.

[9] Singh, M. and Gupta, R.K. (2014) Journal of Fluids, 2014, Article ID: 714150.

[10] Troshkin, O. (2016) Mathematics and Statistics, 4, 47-57. https://doi.org/10.13189/ms.2016.040201

[11] Campos Pinto, M. and Charles, F. (2016) Proceedings and Surveys, 53, 38-48. https://doi.org/10.1051/proc/201653003

[12] Cotter, C.J., Eldering, J., Holm, D.D., Jacobs, H.O. and Meier, D.M. (2016) Journal of Nonlinear Science, 26, 723-765. https://doi.org/10.1007/s00332-016-9317-6

[13] Cubos-Ramírez, J.M., Ramírez-Cruz, Salinas-Vázquez, J.M., Vicente-Rodríguez, W., Martinez-Espinosa, E. and Lagarza-Cortes, C. (2016) Computers \& Fluids, 136, 212-227.

[14] Rehman, K. and Cho, Y. (2016) Water, 8, 212. https://doi.org/10.3390/w8050212

[15] Ogilvie, G.I. (2016) Journal of Plasma Physics, 82, 212. https://doi.org/10.1017/S0022377816000489

[16] Kirtskhalia, V. (2016) Journal of Fluids, 2016, Article ID: 4519201. https://doi.org/10.1155/2016/4519201

[17] Kirtskhalia, V. (2012) Open Journal of Acoustics, 2, 80-85. https://doi.org/10.4236/oja.2012.22009

[18] Kirtskhalia, V. (2013) Journal of Modern Physics, 4, 1075-1079. https://doi.org/10.4236/jmp.2013.48144

[19] Kirtskhalia, V. (2015) Applied Problems of Gas and Hydrodynamics. Academic Publishing "LAMBERT”, Saarbrucken. 